Check for updates

Cite this: Nanoscale, 2020, 12, 23282

\section{Correction: Direct growth of a porous substrate on high-quality graphene via in situ phase inversion of a polymeric solution}

\author{
Yanzhe Qin, ${ }^{a}$ Stephan Koehler, ${ }^{b}$ Yongyou Hu, ${ }^{\star c}$ Yuqing Wu, ${ }^{d}$ Xinwen Peng ${ }^{d}$ and \\ Ming $\mathrm{Ni}^{* a}$
}

DOI: $10.1039 / \mathrm{d} 0 \mathrm{nr} 90261 \mathrm{f}$

rsc.li/nanoscale

Correction for 'Direct growth of a porous substrate on high-quality graphene via in situ phase inversion of a polymeric solution' by Yanzhe Qin et al., Nanoscale, 2020, 12, 4953-4958, DOI: 10.1039/C9NR09693K.

The authors regret their oversight in omitting to attribute the images in Fig. 2a, b, d, e and 4b of this paper, and Fig. S1a, S1b and S1d in the ESI to their original source, a previous publication by the authors in ACS Applied Materials \& Interfaces, which is cited as Ref. 17 in the main article, and Ref. 1 here. Prior to publication, the authors had obtained permission to reproduce these images.

The corrected figure captions for Fig. 2, 4 and Fig. S1 are shown below:

Fig. 2 Characteristics of graphene transferred by IP and ET techniques. (a) Photograph (top view) of a $9 \mathrm{~cm} \times 7 \mathrm{~cm}$ final graphene composite membrane. (b) SEM image shows the integrity of the graphene composite membrane via the IP technique (scale bar is $10 \mu \mathrm{m}$ ). (c) SEM image shows the defects of the graphene composite membrane via the ET technique (scale bar is $10 \mu \mathrm{m}$ ). (d) SEM image of a single finger-like pore covered by integrated graphene on the composite membrane (scale bar is $1 \mu \mathrm{m}$ ). (e) Raman spectrum of the PES raw material, graphene on copper and the PES-graphene membrane. (f) An AFM scanning image shows the topography of a $4 \mu \mathrm{m} \times 4 \mu \mathrm{m}$ graphene composite membrane. a, b, d and e are reproduced with permission from Ref. 1, 2017, the American Chemical Society.

Fig. 4 (a) The SEM image of the PES membrane by the IP techniques using an incorrect formula. Inner stress is formed and it causes film deformation; graphene does not adhere to the PES membrane. (b) The morphologies of the intersection of peeling the PES composite membrane from graphite after coagulation. (c) The SEM image of a PES graphene composite film with a pore size of about $500 \mathrm{~nm}$ with a low pore ratio. (d) The SEM image of a PES graphene composite film with a pore size of about $500 \mathrm{~nm}$ with a high pore ratio, which cause graphene defects. (b) is reproduced with permission from Ref. 1, 2017, the American Chemical Society.

Fig. S1 The SEM images of morphologies of different layers of PES porous substrate via IP techniques. (a) The SEM image of the skin layer that formed within a few seconds after PES solution is immersed into water (scale bar is $10 \mu \mathrm{m}$ ). (b) The SEM image of membrane surface after removal of the skin layer (scale bar is $10 \mu \mathrm{m}$ ). (c) The SEM image of membrane surface (graphene side) after several seconds of oxygen plasma etching (scale bar is $10 \mu \mathrm{m}$ ). (d) Cross-section SEM image of the composite graphene membrane after removal of the skin layer reveals long fingerlike pores that span the membrane (scale bar is $20 \mu \mathrm{m}$ ). Fig S1a, S1b and S1d are reproduced with permission from Ref. 1, 2017, the American Chemical Society.

The Royal Society of Chemistry apologises for these errors and any consequent inconvenience to authors and readers.

\title{
References
}

1 Y. Qin, Y. Hu, S. Koehler, L. Cai, J. Wen, X. Tan, W. L. Xu, Q. Sheng, X. Hou, J. Xue, M. Yu and D. Weitz, ACS Appl. Mater. Interfaces, 2017, 9, 9239-9244.

\footnotetext{
${ }^{a}$ BGI-Shenzhen, Beishan Industrial Zone, Yantian District, Shenzhen, China. E-mail: niming@genomics.cn

${ }^{b}$ Harvard T.H. Chan School of Public Health, Boston, MA, USA

${ }^{c}$ The Key Lab of Pollution Control and Ecosystem Restoration in Industry Clusters, Ministry of Education, School of Environment and Energy, South China University of Technology, Guangzhou Higher Education Mega Centre, Guangzhou 510006, China. E-mail: ppyyhu@scut.edu.cn

${ }^{d}$ State Key Laboratory of Pulp and Paper Engineering, South China University of Technology, Guangzhou 510641, China
} 